\title{
Criminologie
}

\section{La criminalité des affaires et les sentences applicables aux personnes morales}

\section{Marc Brissette}

Volume 15, numéro 1, 1982

Droit et justice

URI : https://id.erudit.org/iderudit/017151ar

DOI : https://doi.org/10.7202/017151ar

Aller au sommaire du numéro

Éditeur(s)

Les Presses de l'Université de Montréal

ISSN

0316-0041 (imprimé)

1492-1367 (numérique)

Découvrir la revue

Citer cet article

Brissette, M. (1982). La criminalité des affaires et les sentences applicables aux personnes morales. Criminologie, 15(1), 77-104.

https://doi.org/10.7202/017151ar d'utilisation que vous pouvez consulter en ligne.

https://apropos.erudit.org/fr/usagers/politique-dutilisation/ 


\section{LA CRIMINALITÉ DES AFFAIRES ET LES SENTENCES APPLICABLES AUX PERSONNES MORALES \\ Marc Brissette*}

Depuis très longtemps, la civilisation occidentale connaît le phénomène de la responsabilité pénale des personnes physiques. Surtout à l'époque oủ morale et droit se confondaient pratiquement. tout comportement individuel dérivant de la norme collective était sévèrement réprimé.

Cette sorte de responsabilité pénale reste encore très importante car même aujourd'hui l'individu, le simple citoyen, à la mention du mot loi, ne pense ni aux contrats, ni aux testaments, ni aux autres questions dont parlent les avocats. Il y a dix chances contre une qu'il pense à la police, car pour lui, la loi veut dire d'abord et avant tout le droit pénal'.

Mais parallèlement à cette importance constante à travers les siècles de la responsabilité pénale des personnes physiques, se développa dans l'évolution de notre civilisation, et plus particulièrement dans l'histoire des droits criminels britannique et canadien, une forme de responsabilité pénale qui s'attache à la répression des actes dérogatoires commis par des personnes morales. En effet, l'omniprésence de la personne morale dans la plupart des activités importantes de nos sociétés contemporaines, et la croissance notable du nombre des crimes qui sont le fait de groupes organisés, rendaient nécessaire la création de cette responsabilité pénale des personnes morales. Cette forme de responsabilité pénale fut reçue en droit canadien au début du siècle, mais ne fut utilisée vraiment couramment devant les tribunaux canadiens que dans la seconde moitié du $\mathbf{x x}^{\mathrm{e}}$ siècle ${ }^{2}$.

Strictement parlant cette forme de responsabilité pénale reconnaît que des actes illégaux peuvent être posés, qui ne sont pas considérés juridiquement comme étant seulement des actes d'indivi-

* Procureur de la Couronne, Cour municipale de Montréal.

1. La responsabilité stricte, document de travail no 2 de la Commission de réforme du droit du Canada, p. 1

2. Pour un historique plus complet de l'avènement de la responsabilité pénale des personnes morales en droit anglais, réfèrer à Deigh, L.H., The Criminal Liability of Corporations in English Law, L.S.E. Research monographs, 2, London, 1969; Brissette, Marc, Les sanctions pénales et les personnes morales. 1978, mémoire de maîtrise en droit, Université de Montréal, p. 18 à 61 pour l'historique en droit anglais, et p. 61 à 6 lf pour l'historique en droit canadien. 
dus, mais comme étant d'abord le fait d'un groupe ayant une personnalité morale dans la poursuite d'une intention commune de ses membres ou d'une fin attribuée à la personne morale lors de sa création, c'est-à-dire comme étant le fait d'une personne morale distincte des personnes qui la composent ${ }^{3}$.

Un juriste, philosophe américain, M. H.N. Seney, dans un savant article sur la désuétude du droit criminel actuel, montre jusqu'à quel point celui-ci n'a pas suivi la réalité changeante de notre époque, et s'en est tenu prudemment à la répression de la criminalité individuelle; on a trop négligé, selon lui, de rechercher les moyens, les concepts et les mécanismes nécessaires à la répression de la criminalité des corps organisés qui est devenue aujourd'hui d'une part la plus importante tant par la quantité de dommages causés à la société en conséquence des infractions que par la quantité d'infractions commises, et qui est devenue d'autre part la plus dangereuse à cause de leurs moyens d'actions décuplés par les puissantes structures de leurs organisations. La position de ces corps organisés est telle qu'elle permet de mettre le simple citoyen en état évident d'infériorité, et d'exercer un pouvoir souvent corrosif sur les institutions politiques et judiciaires dont le rôle fondamental serait pourtant d'assurer cet équilibre des forces par l'égalité devant la loi. Nous arrivons au résultat d'une civilisation qui omet de policer suffisamment les plus importantes de ses forces agissantes ${ }^{4}$.

$\mathrm{Si}$, en effet, avant les lois anglaises sur les Compagnies de 1855 et de 1862, les activités des corporations se limitaient en général aux entreprises de banque, d'assurances, de chemins de fer, de services municipaux, de mines et de manufactures, elles envahirent après ces dates la plupart des autres secteurs de l'économie contemporaines. Tant et si bien que dès 1893 , M. W.S. Gilbert affirmait que l'Angleterre était en voie d'être gouvernée selon le «Joint stock principle ${ }^{6}$.

Au Canada, il en est de même en cette deuxième moitié du $\mathrm{xx}_{\mathrm{r}}$ siècle. Selon M. Raynauld, les compagnies ou sociétés par actions produisaient en 1959, 95,2\% de la valeur totale des expéditions dans

3. Pour une étude des mécanismes d'imputation de la responsabilité pénale à des personnes morales, référer aux mêmes sources que celles citées dans la référence précédente. L.R. 777 .

4. H.W. Seney, Our Criminal Law's Moral Obsolescence (1971), 17 Wayne

5. Hunt, The Development of the Business Corporation in England (1936), p. 145-159, cité par Leigh, The Criminal Liability of Corporations in English Law, L.S.W. Research Monographs 2, London 1969, p. 211.

6. Gilbert, Utopia Ltd., cité par Hunt, o.p. cit., p. 159. 
l'industrie manufacturière et étaient à l'origine de 92,6\% de l'emploi dans ce secteur, malgré qu'elles n'y représentaient que $48,2 \%$ des entreprises ${ }^{7}$. Et à l'intérieur même de ces compagnies, il y a une telle concentration de la production aux mains d'un nombre restreint d'entre elles qu'en 1959, moins de $10 \%$ des établissements $(9,2 \%)$ fabriquaient des produits pour une valeur dépassant 1 million de dollars, et ces $10 \%$ des établissements fabriquaient ensemble $81 \%$ de la production manufacturière au Canada ${ }^{8}$.

Aussi dans ce contexte, devient-il particulièrement pertinent de faire l'inventaire et la description des peines et mesures prévues dans l'ensemble des lois canadiennes et québécoises pour permettre l'application de cette responsabilité pénale à des personnes morales, en d'autres mots de circonscrire le régime des sentences applicables à des personnes morales.

Pour ce faire nous procéderons en deux grandes étapes. Premièrement, il sera nécessaire de faire un inventaire général des sortes de peines et de mesures qui peuvent être utilisées contre les personnes morales pour réagir à la commission d'infractions par cellesci. En' effet, nous n'avons pas voulu limiter cet inventaire aux seules sanctions pénales traditionnelles, mais y inclure toutes les mesures que la loi prévoit à la suite de la commission d'infractions. En ce sens, ces mesures méritent aussi le qualificatif de pénales car le premier but de leur emploi évoqué en cette première section est toujours de contrecarrer le crime ou les effets de celui-ci.

Puis dans une seconde section, nous traiterons essentiellement du registre de gradation de l'amende selon qu'il s'agisse de l'une ou l'autre sorte d'inf ractions. Le législateur a décidé comme règle générale d'imposer la peine d'amende aux personnes morales, et pour bien la décrire il est essentiel de circonscrire les diverses limites du pouvoir discrétionnaire conféré aux tribunaux par la loi pour en déterminer le montant dans chaque cas.

Mais l'ensemble de ces peines aujourd'hui applicables aux corporations, n'ont pas été créées ex nihilo spécialement pour les corporations. Au contraire, le législateur n'a fait en général qu'un strict minimum de modifications législatives pour rendre les lois applicables aux corporations, et dans cette optique il n'a que transposé les peines existantes aux corporations à la seule condition que ce soit

7. André Raynauld, Institutions Economiques Canadiennes. Ed. Beauchemin, Montréal 1964, p. 130.

8. Id., p. 135 . 
physiquement possible ${ }^{9}$.

C'est donc ce simple cheminement d'adaptation des peines existantes aux corporations, qui explique l'omniprésence actuelle de l'amende pour les corporations.

Section 1: Nomenclature des peines et des mesures inscrites dans les lois

Notions générales

On peut décrire globalement l'état actuel du régime des peines prévues pour les personnes morales, tant au Canada qu'au Royaume-Uni, en disant :

... and to this day, the fine remains the only mode of punishment applicable to a corporation (apart of course, from any statutory disqualification or forfeiture $)^{10}$.

En effet, déjà l'article 646 (1) du Code criminel canadien ' ', permettait de remplacer par une peine d'amende toute peine d'emprisonnement dont le maximum prévu est de moins de cinq ans sauf si la loi prévoit un minimum d'emprisonnement, et ce pour toute personne physique ou morale.

Mais l'article 647 vient véritablement préciser le régime général des peines applicables à une corporation, et ce à la fois en ce qui a trait aux actes criminels et aux infractions punissables sur déclaration sommaire de culpabilité.

Art. 647 Nonobstant le paragraphe 646 (2), une corporation déclarée coupable d'une infraction est passible au lieu de toute période d'emprisonnement prescrite comme peine pour cette infraction,

a) d'une amende dont le montant est à la discrétion de la cour, si l'inf raction est un acte criminel, ou

b) d'une amende dont le montant ne doit pas excéder $\$ 1,000$, s'il s'agit d'une inf raction punissable sur déclaration sommaire de culpabilité.

Il ressort entre autres choses de ce texte, que si l'amende peut remplacer l'emprisonnement lorsqu'il est prévu, elle ne dispense nullement la corporation de se soumettre à l'imposition d'autres

9. Pour un historique de ces modifications législatives, on peut se référer à l'article suivant : Lagarde, Irénée. Assignation et procès des corporations en matière pénale (1964), 24 R. du B. 61.

10. G. Williams, Criminal Law. The General Part, 2nd ed., London 1961, 864.

11. Art. 646 (1) Un accusé déclaré coupable d'un acte criminel punissable d'un emprisonnement de cinq ans ou moins peut être condamné à une amende en sus ou au lieu de toute autre punition autorisée, mais un accusé ne doit pas être condamné à une amende au lieu d'un emprisonnement lorsque l'infraction dont il est déclaré coupable est punissable d'une période ninimum d'emprisonnement. 
sortes de peines lorsqu'elles lui sont applicables. Cela est de la plus haute importance, puisque l'efficacité de la sanction pénale à l'égard des corporations repose pour une large part dans la possibilité d'imposer des peines variées et adaptées à chaque cas, et puisqu'on peut s'interroger sur l'utilité actuelle de la peine d'amende à l'égard des corporations.

Aussi des dispositions particulières, éparses à travers les lois, pourront imposer spécifiquement pour certaines infractions, différentes sortes de peines autres que l'amende et l'emprisonnement, comme nous les énumérerons plus loin. Ainsi dans le Code criminel même, on peut donner l'exemple d'une ordonnance spéciale d'interdiction de possession d'arme à feu à l'article 98 (1), C.cr. ou l'exemple d'une peine de révocation d'un certificat ou d'un permis à l'article 106.4 (l) C.cr.

Des dispositions générales s'ajoutent aux dispositions particulières pour faire appliquer à des corporations des peines autres que l'amende ou l'emprisonnement. Ainsi, spécialement les articles 653 (1) et 655 (1) du Code criminel permettent respectivement que des ordonnances soit de dédommagement d'une victime requérante pour perte de biens, ou soit de restitution de biens mal acquis soient émises contre les personnes déclarées coupables d'un acte criminel.

Enfin les articles $115(1)^{12}$ et 116 (1) C.cr. ${ }^{13}$ disposent des cas de contravention à une loi du Parlement du Canada ou à un ordre légal donné par une cour de justice lorsque aucune peine n'est prévue. Les articles 115 (1) et 116 (1) prévoient une peine d'emprisonnement de deux ans à cet égard, ce qui est convertissable en peine d'amende pour les corporations en vertu de l'article 647.

\section{Les peines et mesures visant la réparation}

Traditionnellement en droit criminel britannique, l'obligation de réparer les dommages causés n'entre pas dans les 'sanctions prononcées à l'issue d'un procès criminel, parce que essentiellement cela fait l'objet d'un recours civil distinct de la poursuite pénale, re-

12. Art. 115 (1) À moins qu'une peine ou un châtiment ne soit expressément prévu par la loi, quiconque, sans excuse légitime, contrevient à une loi du Parlement du Canada en accomplissant volontairement une chose qu'elle prétend ou en omettant volontairement de faire une chose qu'elle prescrit, est coupable d'un acte criminel et passible d'un emprisonnement de deux ans.

13. Art. 116 (1) Quiconque, sans excuse légitime, désobéit à un ordre légal donné par une cour de justice ou par une personne ou un corps de personnes autorisé par une loi à donner ou décerner l'ordre, autre qu'un ordre visant le paiement d'argent, est, à moins que la loi ne prévoie expressément quelque peine ou châtiment ou autre mode de procédure. coupable d'un acte criminel et passible d'un emprisonnement de deux ans. 
cours centré en bonne partie sur la preuve de la valeur des dommages. M. Leigh exprime d'ailleurs l'avis qu'une cour criminelle n'est pas compétente pour bien évaluer les dommages ${ }^{14}$. On peut raisonnablement partager son opinion quant à la difficulté de bien évaluer les dommages au cours d'un procès criminel. Et c'est sans doute pour cette raison que même s'il existe à l'article 653 C.cr. une ordonnance de dédommagement que peut émettre une cour criminelle en condamnant un accusé, cette ordonnance se limite aux cas de perte de biens ou de dommage à des biens; car leur valeur peut généralement être déterminée sans trop de problèmes.

Mais ce n'est pas là la seule restriction au champ d'application de cet article 653 C.cr. En effet, en lisant attentivement le texte de cet article ${ }^{15}$, on s'aperçoit qu'il ne peut s'appliquer qu'à la demande de la personne lésée, que si cette demande est faite exactement au moment de l'imposition de la sentence, que si la perte de biens ou les dommages aux biens sont directement causés par la commission de l'infraction et que si cette infraction est un acte criminel. Toutes ces restrictions sont un peu exagérées. M. McLean commente ainsi la restriction selon laquelle la demande de cette ordonnance doit être faite au moment de la sentence :

The application had to be made immediately after conviction, and while this was, no doubt a sensible qualification, it worked considerable injustice in practice. The court might know that the person aggrieved was anxious to apply for compensation and yet, because he was ill, or on holiday, or had not been warned by the police to attend, or had lost his way to the court, the tribunal was precluded from making an order ${ }^{16}$.

Il est à remarquer que le Parlement de Grande-Bretagne a aboli en 1972 la plupart de ces restrictions ${ }^{17}$. Mais ce ne sont là que les restrictions formellement prévues par le texte de loi. S'y ajoutent les restrictions survenant dans la pratique, que la Cour d'appel an-

14. H. Leigh, op. cit., supra note 2, p. 160 . With rare exceptions, the quantum of benefit can only be determined by using a complex costing analysis. Inquiries as to the amount of benefit obtained are not really suited to the summary procedure of a criminal trial. Furthermore the end of criminal punishment is not monetary compensation and there is no reason to permit an exception in the field of corporate crime.

15. Art. 653 (1) C.cr. Une cour qui condamne un individu acusé d'un acte criminel peut, sur la demande d'une personne lésée, lors de l'imposition de la sentence, ordonner que l'accusé paie à ladite personne un montant comme réparation ou dédommagement pour la perte de biens ou le dommage à des biens qu'a subi le requérant par suite de la perpétration de l'infraction dont l'accusé est déclaré coupable.

16. McLean, Ian, Compensation and Restitution Orders, (1973) Crim. L.R., p.4.

17. Criminal Justice Act, (1972) s.1.; cf.I. McLean, loc. cit., 4, 
glaise énonce comme suit et dont nous ne retiendrons pour nos besoins canadiens que les deux dernières :

Only... b) if the victim is prepared to adduce evidence of the loss and c) a determination would not involve investigation of conflicting and perhaps complex stories, should a court consider making an order. If all those criteria cannot be satisfied then the victim should be left to obtain a remedy in ther civil courts $^{18}$.

En fait, l'esprit des articles de loi permettant une ordonnance de dédommagement est assez bien rendu par cette simple phrase de M. Brazier:

Time and again the Court of Appeal (of England) has stressed that the availability of civil law remedies to the victim should not influence the criminal courts. Compensation orders provide quick and simple machinery to be used in straightforward cases: Daly 19.20 .

Il semble que ce soit le fait de devoir s'en tenir à ces "straightforward cases " qui entraîne les restrictions ci-haut énoncées dans R.V. Kneeshaw, dont, entre autres, l'exigence que le montant du quantum demandé soit clair et non litigieux. On a enfin développé la règle selon laquelle on doit tenir compte des moyens financiers de l'accusé, c'est-à-dire de sa capacité à assumer ce dédommagement ou cette réparation du préjudice causé2 1 . On a élaboré cette règle en pensant aux personnes physiques. Devrait-on l'appliquer aux personnes morales afin, par exemple, d'éviter une faillite ou une fermeture de leur entreprise? Si oui, c'est une autre restriction à l'application d'ordonnances de dédommagement qui peut survenir souvent, car dans plusieurs cas, des corporations ne commettent des infractions que pour pouvoir survivre, relancer leurs affaires en mauvais état ou traverser une période creuse.

Et si l'on fait la somme de toutes ces restrictions du texte de l'article 653 et de toutes ces restrictions de fait se dégageant de l'esprit de cette procédure, on peut conclure que dans la grande majorité des causes pénales, notre droit actuel ne permet pas l'obtention d'une telle ordonnance de dédommagement. Mais il faut noter que l'article 655 C.cr. impose à la cour l'obligation de restituer à la personne qui y a droit, les biens obtenus par la perpétration d'un acte

18. Brazier, Rodney, Appellate Attitudes towards Compensation Orders, (1977) Crim. L.R., 712; citant R.V. Kneeshaw (1975) Q.B. 57 (C.A.).

19. R.V. Daly. (1974) I W.L.R. 133 (C.A.).

20. R. Brazier, loc. cit. supra note 17,711.

21. R.V. Oddy, (1974) 2 All E.R. 666 (C.A.); R.V. McKinley (1975) Crim. L.R. 294 (C.A.) 
criminel, mais encore une fois que si cela est possible et facile 22 .

D'autre part, comme nous le verrons plus loin, l'ensemble des autres peines appliquées actuellement aux corporations n'affichent généralement aucun caractère réparateur pour les victimes. Mais, il existe, par exemple dans les lois provinciales du Québec que nous avons spécialement étudiées, des peines d'ordonnances qui sont de nature mi-reparatrice, mi-répressive. Il y a 84 cas d'ordonnances qui furent recensés dans les lois du Québec en vigueur en 1975, ce qui représente $5,3 \%$ des unités d'infractions. Sur ce nombre, on compte 34 ordonnances mandatoires, c'est-à-dire ordonnant de faire quelque chose, 20 ordonnances de démolition, 11 ordonnances de faire exécuter des travaux aux frais du contrevenant, et 5 ordonnances de paiement de dommages-intérêts punitifs. Ces ordonnances sont dans une faible majorité cumulatives avec d'autres peines. Elles ne sont que les seules peines prévues pour une infraction que dans un tiers des cas. Elles visent donc le plus souvent à compléter d'autres peines, et dans un tiers des cas à apporter une solution bien spécifique à une situation donnée 23 .

On peut aussi utiliser le recours extraordinaire qu'est l'injonction, si on en remplit les strictes conditions, et obtenir qu'on ordonne de remettre les choses dans leur état primitif, si cela est possible. Mais ce n'est pas vraiment une sanction pénale.

Voilà donc un arsenal de peines bien pauvres pour remplir cette fonction de réparation, arsenal qui mériterait d'être complété.

\section{Les peines et mesures visant la neutralisation}

Essentiellement on peut utiliser théoriquement dans ce but, soit des peines affectant la personne de la corporation condamnée,

22. Art. 655 (1) C.cr.: Lorsqu'un accusé est déclaré coupable d'un acte criminel, la cour doit ordonner que tous biens obtenus par suite de la perpétration de l'infraction soient rendus à la personne qui y a droit, si lors du procès, les biens se trouvent devant la cour ou ont été détenus de façon à pouvoir être immédiatement rendus à cette personne aux termes de l'ordonnance.

23. Ces statistiques sont tirées d'une recherche sur les infractions dans les lois du Québec que j'ai eu l'occasion de faire de 1975 à 1977 en collaboration avec Me Pierre Robert au Centre international de criminlogie comparée de l'Université de Montréal. Cette recherche consiste essentiellement en un recensement et en une analyse des textes législatifs créant des infractions dans les lois du Québec en vigueur en septembre 1975. Les principales données résultant de cette recherche se retrouvent dans un rapport en deux volumes que $j$ 'ai rédigé et qui est disponible au Centre de documentation de l'École de criminologie de l'Université de Montréal, ainsi qu'au Service de la recherche du ministère de la Justice du Québec qui dispose en plus d'un jeu de cartes de consultation des données pouvant être mises dans un ordinateur. Dans les références ultérieures, je le citerai comme suit : Rapport final de la recherche sur l'infraction dans le droit pénal du Québec, C.I.C.C., Montréal 1977, p. 444 (pour les statistiques sur les ordonnances). 
soit des peines affectant les droits de cette corporation, soit des peines affectant le patrimoine du condamné.

La principale peine neutralisante affectant la personne de la corporation est la dissolution de la corporation. On peut aussi affecter les droits de la corporation pour neutraliser sa possibilité de commettre des infractions en suspendant ou en révoquant ses permis d'opération, dans l'une ou l'autre activité où elle s'est mal conduite, ou toute autre licence, certificat ou droit dont elle a fait un mauvais usage. Et enfin, une peine affectant son patrimoine peut aussi neutraliser les infractions éventuelles d'une corporation, au moyen de la confiscation spéciale ou de la fermeture d'établissement. Ainsi par exemple, le Code criminel prévoit la confiscation des armes à feu dans les cas d'infractions concernant les armes à $\mathrm{feu}^{24}$, et à la Loi des poids et mesures prévoit la confiscation de balances, de poids et mesures n'indiquant pas le bon poids ${ }^{25}$.

Mais voyons d'abord la peine de neutralisation générale par excellence, soit la dissolution de la corporation.

\section{La neutralisation générale}

Il n'existe pas de pouvoir général des juges de juridiction pénale au Canada d'imposer comme peine la dissolution de la corporation à l'issue de procédures pénales. Un tel pouvoir existe peutêtre dans quelques dispositions précises de lois statutaires, mais nous n'en avons pas fait l'inventaire. Mentionnons seulement l'article 230 de la Loi des compagnies du Québec qui permet à un juge de la Cour supérieure d'annuler, sur requête du ministre, les lettres patentes d'une compagnie sans but lucratif pour des motifs d'intérêt public, sans plus de précisions, et les lois des sociétés d'agriculture et des cercles agricoles qui permettent la confiscation de tous les actifs d'une compagnie agissant dans ces domaines d'activité26.

Mais il existe plusieurs cas où on peut obtenir au moyen d'un recours civil la dissolution d'une corporation, pour différentes raisons plus souvent d'ordre civil que criminel. La dissolution se fait dans la province de Québec par la révocation des lettres patentes des corporations ${ }^{2}$.

24. Art. 106 (1) C.cr.

25. S.R.C. (1970), W-7 Art. 45 (4) - 54 (3).

26. Loi des sociétés d'agriculture, (1964) S.R.Q. Ch. 112, art. 69. Loi des cercles agricoles, (1964) S.R.Q. Ch. 113, art. 44.

27. Voir particulièrement les articles 828 à 833 du Code de procédure civile du Québec qui permettraient peut-être de revoquer les lettres d'une corporation utilisée essentiellement pour commettre des infractions. 


\section{Les neutralisations particulières}

Parmi les autres peines pouvant servir les fins de neutralisation du délinquant corporatif, dont nous avons fait mention au début de cette section, il y a les peines d'annulation ou de suspension de permis, licence, etc. Ces peines existent dans plusieurs lois tant fédérales que provinciales et elles peuvent être, selon le cas, appliquées aux détenteurs de permis, soit au moyen de procédures judiciaires pénales, soit par l'exercice du pouvoir discrétionnaire d'un fonctionnaire ou d'un ministre désigné dans la loi. Ainsi dans les lois de la province de Québec, en vigueur en 1975, ces peines affectant un droit ou un privilège autre qu'un emploi ou une fonction, sont utilisées par 12,8\% de tous les textes d'infractions, soit à 205 occasions $^{28}$. Il est possible que tous ces textes d'infractions comportant cette peine ne s'appliquent pas formellement aux corporations, mais souvent ces dernières peuvent être touchées indirectement par ces peines. Si, par exemple, les chauffeurs d'une compagnie de transport perdent leur permis de conduire pour avoir contrevenu aux lois de la circulation en voulant répondre aux exigences de rapidité ou de rentabilité de la compagnie, cette dernière devra remplacer ces chauffeurs en ne pouvant pas nécessairement les renvoyer.

La confiscation de biens généralement meubles, est une peine moins souvent prévue dans les lois et dont on peut penser qu'elle exerce un effet de neutralisation moindre que lorsqu'on interdit un champ d'activité à une corporation en lui enlevant son permis d'opération. Dans les lois de la province de Québec, cette peine de confiscation ne se retrouve que dans 67 textes d'infraction, soit dans $4,2 \%$ d'entre eux ${ }^{29}$.

On peut résumer cette nomenclature des peines en distinguant tout d'abord entre les peines qui peuvent être imposées pour punir toute commission d'une sorte d'infraction ou d'une majorité des infractions par une corporation, et les peines qui ne sont prévues que dans un texte précis pour punir une infraction à ce texte. Ainsi les articles 647a-653-655-115 (1) et 116 (1) du Code criminel sont des dispositions d'application générale déterminant que certaines peines peuvent être imposées pour l'ensemble des actes criminels; l'article 647b fait de même à l'égard des infractions punissables sur déclaration sommaire de culpabilité des lois fédérales. Quant aux infractions aux lois provinciales du Québec et aux règlements municipaux

28. Rapport final de la recherche sur l'infraction dans le droit pénal du Québec, op. cit., supra note 22,426 .

29. Id., 441 . 
de cette province, le régime des peines applicables aux corporations est celui que prévoient les dispositions de chacune de ces lois ou règlements.

Ainsi, les peines d'application générale sont l'amende et l'ordonnance de dédommagement ou de restriction tandis que les peines spécifiques à chaque texte d'infraction peuvent être une amende dont le montant peut varier avec chaque texte, une suspension ou un retrait de permis quelconque, une confiscation de biens ou une ordonnance particulière. Quant aux moyens d'obtenir la dissolution d'une corporation, il s'agit là de mesures spéciales n'ayant pas à proprement parler la nature d'une sanction pénale.

Section 2: Le régistre de la gradation des peines dans les lois

Dans l'appréciation de la gradation des peines prévues pour les corporations, nous nous en tiendrons presque uniquement au cas de l'amende, car c'est surtout elle qui fait l'objet d'une gradation.

Nous scruterons d'abord les limites de la gradation des montants d'amende pour chacune des sortes d'infractions dans les textes directement relatifs aux infractions. Puis, nous nous pencherons sur l'existence d'une atténuation générale apportée à l'ensemble du régistre de gradation des montants d'amende par une disposition de la Loi de l'impôt sur le revenu du Canada.

\section{L'amende pour un acte criminel}

L'examen de l'article 647 C.cr. ${ }^{30}$ permet de constater qu'un juge jouit d'un pouvoir discrétionnaire absolu et illimité quant à la fixation du montant de l'amende tant dans le sens de la sévérité que de la légèreté de l'amende, quand il s'agit d'un acte criminel.

Ce pouvoir discrétionnaire, on ne peut plus large du juge, sur la fixation du montant de l'amende sanctionnant un acte criminel se révèle dans nos annales législatives un exemple rare de confiance presque sans bornes du législateur dans le pouvoir judiciaire. Nous sommes là à la limite du principe de la légalité des peines, si ce n'est au-delà. Car même si le délinquant connaît la nature de la peine qui le menace, il peut difficilement prévoir d'avance s'il subira une peine relativement légère ou une peine très forte. Or n'est-ce pas l'esprit même du principe de la légalité des peines que de permettre de savoir à l'avance si la peine attachée à une infraction sera lourde ou légère? Les effets de cette incertitude sur la dissuasion collective et l'intimidation individuelle des corporations sont probables, sinon presque certains. 
Aussi n'est-il pas étonnant de voir aujourd'hui certaines initiatives législatives ou même réglementaires réagir fortement à cela. Par exemple, dans une loi du Parlement fédéral, les entreprises reconnues coupables d'avoir exploité les consommateurs seront passibles d'amendes pouvant aller jusqu'à un million de dollars, en vertu d'amendements à la Loi sur les coalitions qui ont reçu l'approbation de principe aux Communes. Le député néo-démocrate, John Rodriguez a déclaré à ce sujet que les amendements envisagés étaient nécessaires, car les sanctions actuelles ne constituaient rien moins qu'un encouragement aux compagnies de poursuivre des tactiques malhonnêtes telles que l'entente sur les prix. Actuellement, la peine de prison maximale est de deux ans et l'amende est laissée à la discrétion des tribunaux.

En effet, contrairement à ce que l'on peut croire au premier abord, le fait de laisser le montant de l'amende à la discrétion des tribunaux ne semble pas les inciter à donner de très fortes amendes. mais les porterait plutôt à se méfier et à chercher à se défendre contre leur propre pouvoir discrétionnaire en le limitant dans les faits. Tandis que le fait de fixer un maximum d'amende élevé dans la loi les légitimera davantage d'imposer des amendes plus élevées puisqu'ils seront plus sûrs ainsi d'agir conformément à l'intention du législateur. En d'autres termes, même si ce projet d'amendement législatif paraît théoriquement passer d'un montant d'amende illimité à un montant d'amende limité à un maximum de un million de dollars, en fait on passe d'une amende moins sévère à une amende plus sévère, ce que tend à indiquer le propos du député néo-démocrate.

D’autre part, il résulte inévitablement de ce pouvoir discrétionnaire illimité une très grande disparité dans le degré de sévérité des sentences à l'égard des corporations, ce qui n'est pas pour favoriser le sentiment que justice a été rendue. Cela discrédite le système d'administration de la justice criminelle à la fois aux yeux des corporations, et aux yeux de ceux qu'on veut protéger des activités délictueuses des corporations.

Cependant ce pouvoir discrétionnaire ne peut aller jusqu'au point de n'infliger aucune peine. En effet, le législateur à l'article 662.1 C.cr exclut expressément la corporation de la possibilité de bénéficier d'une libération conditionnelle ou inconditionnelle. $\mathrm{Ce}$ qui entraîne qu'une fois déclarée coupable, la corporation doit être condamnée à une peine quelque faible qu'elle soit. Mais il faut noter que théoriquement le tribunal ne peut condamner une corporation à 
une peine d'amende trop faible puisque l'amende dont il s'agit à l'article 647 C.cr. n'est pas une simple amende ordinaire mais une peine devant tenir lieu d'emprisonnement, lequel est une des peines les plus sévères dans la hiérarchie habituelle du législateur.

Enfin, les tribunaux tiennent compte des condamnations antérieures d'une corporation dans la détermination du montant de l'amende, et ce en l'en avisant lorsqu'elle a comparu, et sans avoir besoin de l'en aviser lorsqu'elle n'a pas comparu ${ }^{31}$.

Il ressort donc essentiellement des précédentes considérations que le pouvoir discrétionnaire sur le montant de l'amende sanctionnant l'acte criminel d'une corporation, conféré aux juges par l'article 647 du Code criminel, est trop grand du fait qu'il n'y ait de limite ni maximale, ni minimale et que cela diminue en fin de compte la sévérité et l'efficacité des peines d'amende alors imposées.

L'amende pour une infraction punissable sur déclaration sommaire de culpabilité

Nous étudierons successivement ici, la peine d'amende prévue par l'article $647 \mathrm{~b}$ C.cr. en remplacement de la période d'emprisonnement prescrite comme peine dans les textes d'infractions et la sévérité de l'ensemble des peines d'amende dans les lois statutaires.

L'article $647 b$ du Code criminel - Après avoir considéré l'ampleur du pouvoir discrétionnaire sur la sévérité des sanctions des actes criminels des corporations, à quel contraste frappant ne sommes-nous pas exposés quand on regarde le maximum de mille dollars prévu en remplacement de l'emprisonnement pour les infractions sommaires de ces mêmes corporations, ce qui représente seulement le double de ce qui est prévu à l'article 722 (1) C.cr. pour punir les infractions sommaires commises par des personnes physiques? Car on peut penser que les actifs des corporations comparativement aux actifs des particuliers peuvent en moyenne ne pas représenter cette proportion.

Mais on peut se demander à la lecture de l'article 647 et surtout des mots « au lieu de toute période d'emprisonnement prescrite comme peine pour cette infraction ", s'il serait permis au tribunal de donner à une corporation pour une infraction punissable sur déclaration sommaire de culpabilité, en plus du montant maximal

31. Art. 592 (2) : Lorsque, en conformité de l'article 551, la cour procède au procès d'une corporation accusée qui n'a pas comparu, ni plaidé à l'égard d'un acte d'accusation, la cour peut, si l'accusée est déclarée coupable, s'enquérir des condamnations antérieures, que l'accusée ait été avisée ou non qu'une plus forte peine serait demandée de ce fait. 
de $1000 \$$ d'amende prévue par l'article 647 au lieu de la peine d'emprisonnement, la peine d'amende qui est déjà nommément prévue au texte de l'infraction dont elle est inculpée. En d'autres termes, si une infraction est poursuivable comme infraction punissable sur déclaration sommaire de culpabilité et a comme peines l'amende et l'emprisonnement, le montant maximal d'amende qu'un tribunal puisse imposer à une corporation pour cette infraction, est-il la somme de $1000 \$$ et du montant maximal de l'amende prévue pour cette infraction, ou est-il de $1000 \$$ ? Il faudrait que les tribunaux tranchent cette question, mais il semble qu'une interprétation littérale du texte de l'article 647 C.cr. permette d'ajouter l'amende prévue à un texte d'infraction, au maximum de $1000 \$$ qui est une peine qui ne fait que remplacer l'emprisonnement et non l'amende cumulative, ni les autres peies d'un texte d'infraction.

Ainsi l'on admet l'interprétation littérale, on peut dire que l'article 647 C.cr. ne fait que disposer de la façon de remplacer la peine d'emprisonnement par une autre peine applicable à une corporation, et ne régit pas le montant de l'amende qui peut être prévue dans le texte même de l'inf raction cumulativement ou alternativement avec l'amende tenant lieu de l'emprisonnement. De telle sorte qu'on puisse soutenir qu'aucune disposition générale au Code criminel empêche, pour sanctionner une infraction punissable sur déclaration sommaire de culpabilité commise par une corporation, ni d'imposer la peine d'amende prévue au texte de l'infraction même si elle est supérieure à $1000 \$$, ni d'avoir une somme de l'amende prévue au texte et de l'amende remplaçant l'emprisonnement qui dépasse $1000 \$$.

Mais même si à l'occasion, on peut ajouter la peine d'amende prévue au texte, au $1000 \$$ de l'article $647 \mathrm{~b}$ C.cr., cette peine d'amende risque d'être bien légère par rapport aux actifs et aux profits d'un grand nombre de corporations.

Les amendes dans les lois statutaires - Avant de s'interroger sur la sévérité des peines d'amende en tant que telles, il importe de se donner une idée de l'importance quantitative de ces peines. La peine d'amende est utilisée à l'intérieur des lois du Québec, dans $76 \%$ de tous les textes d'infractions qui sont plus de 1560 . Elle est donc la sanction pénale par excellence dont on fait un usage presque constant dans les lois pénales du Québec. Les autres peines ne semblent survenir que dans les cas où vraiment la seule peine d'a- 
mende ne suffirait pas à faire appliquer la $l o i^{32}$.

Mais on peut aussi se demander quelle proportion des textes d'infractions des lois québécoises est applicable à des corporations. À la lecture des lois on découvre qu'environ $65 \%$ des infractions peuvent vraisemblablement être commises par des personnes morales, tandis que $32 \%$ des infractions ne peuvent être commises que par des personnes physiques. Cela arrive presque à la proportion de $2 / 3,1 / 3$, ce qui signifie qu'environ 1 millier de textes d'infractions peuvent s'appliquer à des corporations. Mais si 32\% des unités d'infractions sont réservées exclusivement aux personnes physiques, on constate que seules $10,2 \%$ des unités d'infractions sont réservées exclusivement aux personnes morales. Il y aurait donc $54,6 \%$ des unités d'infractions qui peuvent être commises soit par des personnes physiques, soit par des personnes morales indifféremment. Parmi ces dernières, $7 \%$ de toutes les unités d'infractions prévoient expressément pouvoir s'appliquer à des personnes morales ${ }^{33}$. Donc, les cas d'infractions où rien n'empêche qu'elles soient perpétrées par une personne morale représentent $47,7 \%$ des unités d'infractions.

Cela ne signifie nullement que l'on doive considérer le reste des infractions comme ne pouvant pas être commises par les personnes morales. Il n'est nul besoin que le législateur s'exprime expressément à ce sujet dans chaque texte d'infractions. Une abondante jurisprudence confirme cette proposition. Des corporations ont multes fois été condamnées selon des textes qui ne prévoyaient que l'objet de l'infraction et la sanction pénale.

Et parmi les 10,2\% des textes d'infractions ne s'appliquant qu'à des personnes morales, on remarque les proportions suivantes.

Il n'y a aucune infraction consacrée aux corporations professionnelles prises collectivement ou individuellement. Par contre 19 infractions ne s'appliquent qu'à une corporation municipale et 57 infractions ne s'appliquent qu'à une personne morale en particulier ou qu'à des personnes morales ayant une activité particulière. Il y a donc $4,8 \%$ des unités d'infractions ne régissant qu'une personne morale en particulier ou à un genre de personnes morales en particulier, tandis que $5,4 \%$ des infractions expriment clairement ne s'appliquer qu'aux personnes morales en général ${ }^{34}$.

Ainsi, la personne morale est un sujet de droit pénal non né-

32. Rapport final de la recherche sur l'infraction dans le droit pénal du Québec, op. cit., supra note 22,383 .

33. Rapport final de la recherche sur l'infraction dans le droit pénal du Québec, op. cit., 199-200. 
gligeable vis-à-vis les lois provinciales du Québec, et on peut maintenant avoir la certitude qu'il vaut la peine de tenir compte de la sévérité des peines de ces lois.

Les montants des amendes dans les lois du Québec s'échelonnent de $1 \$$ à plus de $50000 \$$. Il existe plusieurs formes d'expression des montants d'amende dans les lois : des montants fixes, des montants maximaux, des montants à la fois minimal et maximal et de façon rarissime quelques montants minimaux seulement.

Dans l'ensemble des données recueillies sur la gradation des montants d'amendes ${ }^{35}$, on constate que les montants d'amende fixe et les montants d'amende minimale sont en très grande majorité de moins de $500 \$$.

On peut cependant remarquer que plus de la moitié des maximums d'amende sont de $500 \$$ et plus, que 157 d'entre eux sont de $5000 \$$ et plus et que quatre d'entre eux sont de $50000 \$$ et plus. Mais ces maximums élevés sont sans doute la conséquence obligatoire de l'existence de 122 cas de minimums d'amende de $500 \$$ et plus.

Ainsi, contrairement aux inf ractions punissables sur déclaration sommaire de culpabilité des lois fédérales, les montants d'amende des lois du Québec comportent des maximums assez souvent supérieurs à $1000 \$$, même s'il s'agit d'infractions poursuivables selon la Loi des poursuites sommaires du Québec ${ }^{36}$.

Tableau de regroupement des montants d'amende

\begin{tabular}{lccc}
\hline & Amende fixe & Amende minimale & Amende maximale \\
\hline 1\$ à $50 \$$ & 83 & 205 & 130 \\
$50 \$$ à $100 \$$ & 16 & 57 & 51 \\
$100 \$$ a $200 \$$ & 28 & 93 & 111 \\
$200 \$$ a $500 \$$ & 23 & 113 & 149 \\
$500 \$$ a $1000 \$$ & 12 & 91 & 125 \\
$1000 \$$ à $5000 \$$ & 11 & 24 & 182 \\
Plus de $5000 \$$ & 1 & 7 & 157 \\
\hline
\end{tabular}

De même façon dans la réglementation municipale, on trouve plusieurs exemples d'accroissement notable de la sévérité des peines. Ainsi un simple règlement municipal de la Communauté urbaine de Montréal sur l'assainissement de l'air ${ }^{36}$ créant des infractions qui devraient être théoriquement hiérarchiquement bien inférieures

34. Id.,199-200.

35. Rapport final de la recherche sur l'infraction dans le droit pénal du Québec, op. cit., supra note 22,400 .

36. (1964) S.R.Q. Ch. 35. 
à des infractions sommaires du Code criminel, comporte des amendes qui ont été ainsi haussées par rapport à l'ancien règlement : l'amende maximale pour une première infraction passe de $500 \$$ à $10000 \$$. Pour une infraction subséquente, l'amende minimale est de $500 \$$ et l'amende maximale est de $20000 \$$.

C'est donc que dans la détermination de la sanction pénale, on ne s'est pas d'abord attaché à la qualification formelle de l'infraction qui n'est qu'une simple contravention à un règlement municipal, mais bien plutôt à l'efficacité de la peine eu égard à l'importance du sujet qu'elle cherche à sanctionner aux yeux des citoyens et à la grosseur des corporations qui devront s'y soumettre. Pour un simple règlement municipal, on est déjà bien loin du montant maximal de $1000 \$$ d'amende prévu pour les infractions sommaires à l'article 647 C.cr.

Enfin, dans les lois tant fédérales que provinciales, il arrive que pour une même infraction l'on prévoie un montant d'amende plus élévé, et parfois beaucoup plus élevé, pour une corporation que pour une personne physique. Ainsi, dans l'ensemble des lois de la Législature du Québec en vigueur en 1975, on retrouve 108 infractions $^{38}$ réparties dans 34 lois qui varient la peine selon qu'elle s'applique à une corporation ou à une personne physique. Une loi provinciale comme la Loi sur les valeurs mobilières ${ }^{39}$ comporte des maximums d'amende pour les corporations qui sont de plus de $50000 \$$.

De même façon, beaucoup de textes d'infractions varient la peine selon qu'il s'agit d'une première infraction ou d'une infraction subséquente. Ainsi dans les lois du Québec, $20 \%$ des textes d'infractions prévoient une peine plus sévère dans les cas de récidive ${ }^{40}$. Mais il n'en reste pas moins que la grande majorité des montants fixes ou maximaux d'amende ne dépassent pas $1000 \$$.

Donc la sévérité de ces peines d'amende est, presque dans la totalité des cas, limitée par un montant maximal d'amende inscrit soit dans une disposition d'application générale comme l'article $647 \mathrm{~b}$ C.cr., soit dans chaque disposition d'une loi statutaire provinciale ou fédérale, créant une infraction. Or, dans l'un ou l'autre cas, les montants maximaux ou fixes de ces amendes s'avèrent générale-

37. Règlement no 44 de la C.U.M.

38. Id., 547. Rapport final de la recherche sur l'infraction dans le droit pénal du Québec, op. cit. supra note 22,547.

39. S.R.Q. (1964) Ch. 274.

40. Rapport final de la recherche sur l'infraction dans le droit pénal du Québec, op. cit., supra note 22,453 a 478 . 
ment assez faibles, comme nous l'avons constaté.

Car comme l'évolution de la vie commerciale et industrielle de l'Amérique du Nord s'est faite rapidement et même de façon accélérée, il est naturel que les montants d'amende inscrits dans une loi, le fussent selon les standards économiques de l'année pendant laquelle cette loi fut adoptée, et selon les besoins de répression de ce temps à l'égard de l'objet de la loi. Or, un grand nombre de lois n'ont pas vu leurs montants d'amende modifiés, bien qu'elle datent de plusieurs décennies, ou même du début du siècle. C'est un peu ce que constate, M. Dershowitz aux États-Unis.

The criminal fine as presently administered against the endocratic corporation guilty of acquisitive crime is totally ineffective as a profit diminishing sanction. Its ineffectiveness is primarily attributable to the exceedingly low quantitative maximum penalties permitted by governing statutes. These statutes were generally enacted at about the turn of the twentieth century and the maximum fines then established have, in many cases, remained unchanged despite an enormous growth in corporate earnings ${ }^{4}$.

Il n'hésite pas même à qualifier de cause la plus importante de l'inefficacité de la peine d'amende, la faiblesse des montants d'amende prévus dans les lois. Aussi ne faut-il pas s'étonner qu'un projet de loi sur la réforme du droit criminel qui sera soumis au Congrès des États-Unis propose que le montant d'amende maximal imposable à une corporation pour un acte criminel relevant du gouvernement fédéral, passe de $50000 \$$ à $500000 \$^{42}$.

Evidemment, quand on parle ici de la légèreté des peines d'amende prévues dans les lois à l'égard des corporations, il s'agit d'une légèreté de la peine comparativement d'abord à l'ampleur des fruits du crime quand il y a lieu, et ensuite comparativement au chiffre d'affaires et aux profits de la corporation. L'importance de la peine d'amende appliquée à une corporation ne dépend pas du montant nominal de cette amende, mais du taux de la fraction que représente l'amende par rapport aux éléments ci-haut mentionnés. C'est en ce sens d'ailleurs, que la Commission de réforme du droit du Canada suggère de repenser la formule de fixation du montant

41. Dershowitz, A.M. Some Observations on the Meaning of Corporate Criminal Liability, excerpts from a paper submitted by the author during his third term as a student in the Yale Law School, 1961.

A13.

42. "A Bill would overhaul U.S. laws", The Montreal Star, 27 janvier 1978, 
de l'amende imposée à une corporation ${ }^{43}$.

Car il devient évident que si un montant donné d'amende peut avoir un certain effet sur une petite compagnie, il risque de représenter moins que rien aux yeux des grosses corporations. M. Dershowitz affirme ainsi que l'effet d'un montant qui paraît assez élevé, soit $50000 \$$ et qui est le montant maximal prévu dans la loi américaine Sherman sur les coalitions, est à peu près nul à cause de sa disproportion par rapport aux profits illégalement touchés par les perpétrations d'infractions à cette $\operatorname{loi}^{44}$.

This figure, however is also less than adequate when one realizes that the justice department seeks criminal remedies only in "flagrant " cases where the amount of profits involved is frequently a substantial multiple of the $\$ 50000$ maximum or the $\$ 13000$ average fine imposed under the amended statute. The General Electric case provides a vivid example. Although the government called this \& the largest of all criminal antitrust cases " and the indictment covered almost $\$ 7$ billion of fixed prices, the average fine imposed upon the corporate defendants on each count mounted to $\$ 16,550$ and the maximum fine of $\$ 50,000$ was imposed but once out of the 159 sentences.

Ainsi, si on jette un coup d'œil sur l'ampleur des actifs de quelques-unes des plus grosses corporations américaines, il devient évident que le montant actuel des amendes ne peut guère les toucher. ${ }^{45}$

Pourtant, une très grande part des poursuites intentées contre des corporations visent à sanctionner la commission d'infractions punissables sur déclaration sommaire de culpabilité. C'est en effet, aux infractions réglementaires qui régissent leurs activités particulières, souvent techniques et spécialisées, que les corporations contreviennent le plus souvent et non pas aux infractions plus générales. En fait, c'est aussi un choix du législateur de régir les corporations au moyen de lois et surtout de règlements très élaborés sur chaque aspect de la vie commerciale et industrielle plutôt qu'au moyen des quelques grandes normes du Code criminel. M. Leigh

43. "Responsabilité pénale et conduite collective", Document de travail no 16 de la Commission de réforme du droit du Canada, Information Canada, Ottawa, 1976, p.43: 4...nous pourrions trouver à l'égard de la Compagnie une formule qui égaliserait la marge de perte subie par celle-ci au moyen d'une amende qui tiendrait compte de facteurs tels que les profits, l'ensemble des actifs et la faculté de faire porter par d'autres le poids de l'amende. "

44. Dershowitz, A.M., op.cit, supra note 38.

45. Davids, loc. cit. supra note, 525. Considérons les chiffres du bilan de 4 grosses compagnies américaines. 
Données représentatives de grandeur de quatre corporations américaines (tel qu'au 31 déc. 1965)

\begin{tabular}{|c|c|c|c|c|}
\hline & Amer. Tel. \& Tel. & Con. Édison & Gen. Motors & Gulf Oil Corp. \\
\hline $\begin{array}{l}\text { Unités } \\
\text { en service }\end{array}$ & $\begin{array}{l}75,866,254 \\
\text { téléphones } \\
\text { en service }\end{array}$ & $\begin{array}{l}4,367,020 \\
\text { compteurs } \\
\text { en service }\end{array}$ & $\begin{array}{c}7,278,131 \\
\text { véhicules } \\
\text { vendus en } 1965\end{array}$ & $\begin{array}{c}2,082,121 \\
\text { compte } \\
\text { journalier }\end{array}$ \\
\hline Employés & 795,294 & 23,863 & 734,600 & 55,200 \\
\hline $\begin{array}{l}\text { Total des } \\
\text { dépenses }\end{array}$ & $\$ 32,818,689,000$ & $\$ 3,387,006,500$ & $\$ 11,478,546,590$ & $\$ 5,210,833,000$ \\
\hline $\begin{array}{l}\text { Revenu } \\
\text { brut } \\
\text { en } 1965\end{array}$ & $\$ 11,061,783,000$ & $\$ 840.240,274$ & $\$ 20,733,982,295$ & $\$ 4,185,253,000$ \\
\hline $\begin{array}{l}\text { Pourcentage } \\
\text { d'augmenta- } \\
\text { tion } \\
\text { du revenu } \\
\text { brut depuis } \\
1960\end{array}$ & $\begin{array}{c}40 \% \\
(\$ 7,920,454,000)\end{array}$ & $\begin{array}{c}28 \% \\
(\$ 655,812,826)\end{array}$ & $\begin{array}{c}63 \% \\
(\$ 12,735,999,681)\end{array}$ & $\begin{array}{c}32 \% \\
(\$ 3,212,205,000)\end{array}$ \\
\hline Source & $\begin{array}{l}\text { Rapport annuel } \\
\text { daté du } 16 \text { fév. ' } 66 \\
\text { p. } 25-28\end{array}$ & $\begin{array}{c}\text { Rapport annuel } \\
\text { daté du } 23 \text { fév. '66 } \\
\text { p. } 26-31\end{array}$ & $\begin{array}{c}\text { Rapport annuel } \\
\text { daté du } 14 \text { fév. ' } 66 \\
\text { p. } 28-36-7\end{array}$ & $\begin{array}{l}\text { Rapport annuel } \\
\text { daté du } 16 \text { mars } 66 \\
\text { p. } 2.34-35\end{array}$ \\
\hline
\end{tabular}

constate cette grande incidence d'inf ractions réglementaires.

One of the striking features disclosed by an examination of the cases has been the low incidence of decisions relating to what might be called traditional crimes. No English case has yet imposed liability for crimes of violence or for crimes against the state. In practice liability generally relates to certain types of commercial fraud or violations of regulatory legislation $^{46}$.

Et la commission de ces infractions sommaires par les corporations n'entraînent pas nécessairement moins de dommages sociaux que la commission des actes criminels moins nombreux et qui ne diffèrent souvent des infractions sommaires que par leur caractère plus normatif ${ }^{4}$.

Malgré cela, l'on doit se contenter d'un régistre restreint de montants d'amende pour tenter de sanctionner les infractions sommaires de la plus grande corporation avec la même sévérité que cel-

46. L.H. Leigh, op. cit., supra note 2. 52.

47. H.W. Seney, Harm. Danger and Dangerousness in Our Criminal Law (1971) 17 Wayne L.R.. 1111-1112. 
les de la plus petite corporation. De plus, dans la réalité, on doit constater qu'en dépit du fait que les montants maximaux d'amende soient peu élevés, la moyenne des montants d'amende imposés est bien inférieure à ces montants maximaux. On peut en donner quelques exemples en considérant successivement des cas d'application pendant une période donnée du règlement 44 de la Communauté urbaine de Montréal sur l'assainissement de l'air et des lois canadiennes sur les aliments et drogues sur les poids et mesures sur l'emballage et l'étiquetage des produits de consommation et sur les pêcheries.

En vertu du règlement sur l'assainissement de l'air, MM. Hétu et Duplessis nous donnent les statistiques suivantes pour la période s'étendant du $1^{\mathrm{er}}$ avril 1970 au $1^{\mathrm{er}}$ juin 1976.

Sur 833 condamnations faisant abstraction qu'il s'agisse d'une première infraction ou non, nous pouvons voir que les juges ont eu tendance à imposer des amendes se situant entre $\$ 25$ et $\$ 49$ et plus près de $\$ 25$ que de $\$ 49$; ceci correspond au minimum fixé par la loi pour une première infraction. À 109 reprises l'amende se chiffra entre $\$ 100$ et $\$ 199,30$ fois entre $\$ 200$, et $\$ 299,10$ fois entre $\$ 300$ et $\$ 399,2$ fois entre $\$ 400$ et $\$ 499$, 14 fois entre $\$ 500$ et $\$ 999$, et 12 fois les amendes imposées par les Cours municipales dépassèrent le $\$ 1000$.

La moyenne des amendes imposées par les juges municipaux du territoire de la C.U.M. pour les 833 chefs d'accusation avec plaidoyer ou verdict de culpabilité s'élève à $\$ 9.45$ pour l'ensemble de la période de 1970 à 1976 . À Montréal, la moyenne de l'amende par chef d'accusation se chiffre à \$68.81 alors que dans les villes de banlieue, les juges municipaux se sont montrées plus sévères puisque la moyenne par chef d'accusation est de $\$ 154.93^{48}$.

Mais ce n'est là qu'une moyenne générale des montants d'amende imposés. Car en regardant la liste des corporations condamnées plus d'une fois en vertu de ce règlement, on remarque que si un certain nombre d'entre elles ont pu recevoir comme peine, une amende de plus de $100 \$$, la majorité des corporations ainsi condamnées ne se voient imposer qu'une amende de $50 \$$ et moins, et le plus souvent de $25 \$$. Et même les corporations recevant comme peine une amende de $1000 \$$ ou un peu plus, sont d'une taille telle et ont un tel volume d'affaires et de profits, que cette amende risque d'être encore

48. Hétu, J. et Duplessis, Y. La pollution de l'air et les cours municipales du territoire de la Communauté urbaine de Montréal, 1975, R.J.T. 328. 
plus imperceptible pour ces corporations. ${ }^{49}$

Nous pouvons examiner, le rapport du ministère fédéral des Consommateurs, sur les condamnations obtenues pendant une période de trois mois se situant bien en dehors de la période de vacances judiciaires, soit entre le premier janvier et le 31 mars 1977, en vertu des dispositions des trois lois suivantes, parmi les plus importantes dans la régie de la vie commerciale : la Loi sur les aliments et drogues ${ }^{50}$, la Loi sur les poids et mesures ${ }^{51}$ et la Loi sur l'emballage et l'étiquetage des produits de consommation ${ }^{52}$.

Pour ces trois lois, pendant trois mois, quarante-quatre entreprises ont été reconnues coupables à travers tout le Canada, dont huit corporations au Québec. Sur les 44 condamnations représentant 109 chefs d'accusations et un total de $38250 \$$ d'amende, 10 ont été prononcées en vertu de la Loi des aliments et drogues, 28 en vertu de la Loi sur les poids et mesures, et six en vertu de la Loi sur l'emballage et l'étiquetage des produits de consommation.

Sur les huit corporations condamnées au Québec, deux le furent en vertu de la Loi des aliments et drogues, trois en vertu de la Loi sur l'emballage et l'étiquetage et deux en vertu de la Loi sur les poids et mesures. Trois de ces corporations eurent une peine d'amende de $3000 \$$, deux d'entre elles une peine de $500 \$$ à $800 \$$, et les

49. Ibid. Font partie du club du $1000 \$$ d'amende et plus :

- Budget Fuels Inc., C.M. Montréal, no 34-2347, 17 juillet 1975 (J. Stalker), $1500 \$$ (Art.14).

- Budget Fuels Inc., C.M. Montréal, no 34-2348, 17 juillet 1975 (J. Stalker), $1500 \$$ (Art.14).

- Budget Fuels Inc., C.M. Montréal, no 34-2349, 17 juillet 1975 (J. Stalker), $1500 \$$ (Art.14).

- Budget Fuels Inc.. C.M. Montréal, no 34-2350, 17 juillet 1975 (J. Stalker), $1500 \$$ (Art.14).

- Budget Fuels Inc., C.M. Montréal, no 34-2351, 17 juillet 1975 (J. Stalker), $1500 \$$ (Art.14).

- Longueuil Meat Exporting Co. Ltd., C.M. Montréal, no 36-0079 1976 (J. Quinlan), 1000 (Art.5).

- Petrofina Canada Ltd., C.M. Pointe-aux-Trembles, no 74-1278, 9 décembre 1974 (J. Dansereau), $1000 \$$ (Art.4).

- Compagnie Internationale de Papier du Canada, C.M. Pointe-aux-Trembles, no 74-1386, 11 décembre 1974 (J. Dansereau), l $000 \$$ (Art.4).

- Petrofina Canada Ltée, C.M. Pointe-aux-Trembles, no 75-0059, 13 mars 1975 (J. Dansereau), 1500 (Art.4).

- Petrofina Canada Ltée, C.M. Pointe-aux-Trembles, no 76-760, (J. Tessier), $1000 \$$ (Art.4).

50. S.C.R., (1970) Ch. F-27.

51. S.R.C. (1970) Ch. W-7.

52. S.C., (1970-71-72) Ch. 41. 


\section{trois autres une peine de $200 \$$ et moins $^{53}$.}

D'autre part, dans la liste des condamnations obtenues contre des corporations au Canada pour des infractions aux dispositions sur la publicité trompeuse ${ }^{54}$, on remarquera que dans cinq cas sur six, l'amende est de $500 \$$ peu importe qu'il s'agisse du Restaurant chez Bou Bou à Jonquière ou de Simpsons-Sears Ltd. de Toronto, même si l'infraction a pu de beaucoup profiter davantage au second

53. "Infractions sur des produits de consommation, 44 entreprises condamnées ", La Presse, mercredi le 8 juin 1977, P. E14.

1) en vertu de la loi des aliments et drogues:

- Produits Blanchet Inc., 468, rue Saint-Roch, à Roch Forest : composition de la margarine, amende : $3000 \$$;

- Ferme Trudel Inc., 555 boul. Laflèche à Hauterive, lait, quantité insuffisante, amende : 200 ;

2) en vertu de la loi sur l'emballage et l'étiquetage:

- Le Rhéaume, 286, lère Avenue au Lac Saint-Charles, substitution de poisson. amende : $500 \$$;

3) en vertu de la loi sur les poids et mesures:

- La Boustifaille (Claude Lapointe), 690, rue Laure à Sept-Iles, viande, poids insuffisant, amende $150 \$$;

- Sobey's Stores Lts., 190 ouest, rue Principale à Chandler, viande, poids insuffisant, amende : 100 \$;

- Garage Belisle et Fils, auto Ltée., Mont-Joli, falsification d'odomètre, amende : $3000 \$$

- Christin Automobile Inc., Montréal, falsification d'odomètre, amende : $800 \$$;

- Pasquale de Filipes Auto Inc., Montréal, falsification d'odomètre, amende : $3200 \$$.

54. Liste donnée par un rapport du ministère fédéral de la Consommation rendu public en janvier 1978; ce qui fait présumer qu'il s'agit des condamnations prononcées lors du ou des mois précédents.

- "Firms Ads Draw Fines ", The Montreal Star, Janvier 1978.

- Pour publicité erronée, une amende de $500 \$$ a été imposée à Jay Norris Corporation de Montréal. Jean-Claude Héroux a reçu une sentence suspendue pour avoir annoncé un Jardin Japonais de huit variétés d'arbustes rabougris quand il a été démontré qu'au lieu de vendre des arbustes qui grandissent, ils vendaient huit graines plantées individuellement.

- Simpsons-Sears Ltd. de Toronto a reçu une amende de $500 \$$ pour avoir annoncé des jouets dans un catalogue de soldes en indiquant qu'ils sont fabriqués par une compagnie bien spécifique. L'enquête démontra qu'ils avaient inclus des jouets fabriqués par d'autres compagnies que celle désignée dans leur catalogue et que ces jouets étaient de qualité inférieure que ceux annoncés.

- Le Royaume du Silencieux du Saguenay Inc. de Jonquière, Qué., reçut une amende de $500 \$$ pour avoir annoncé des silencieux avec une garantie à vie, quand il a été démontré que la garantie n'était pas valable pour les silencieux posés sur les voitures de marque ètrangère.

- Une amende de $500 \$$ a été imposée au Restaurant chez Bou Bou Inc., de Jonquière, Qué. pour avoir annoncé des livraisons gratuites, quand en réalité on percevait des frais de livraison.

- Deux compagnies de Winnipeg ont annoncé un dispositif de haute fréquence connu sous le nom de "Buzz-Off " pour tenir à distance les moustiques. Ce dispositif ne fonctionnant pas ils reçurent une amende de $500 \$$.

- Dockside Marine Ltd. de Kelowna, en Colombie Britannique reçut une amende de $125 \$$ pour avoir annoncé le plus gros choix de bateaux ainsi que la plus grande exposition intérieure de Okanagan, quand il a été démontré que cette publicité était mensongère. 
qu'au premier.

Enfin, Mlle Hélène Dumont nous donne relativement à l'application de la loi sur les pêcheurs, les chiffres suivants :

Encore plus saisissantes sont les statistiques indiquant la moyenne de l'amende imposée pour une violation quotidienne de la loi des pêcheries. L'introduction de substances délétères dans les eaux poissonneuses entraînent en vertu de cette loi une amende de $5000 \$$, par jour de violation; or en 1969, l'amende moyenne est de $10 \$$ par jour; en 1971, elle se situe autour de $14 \$ 55$.

\section{La possibilité de récupération fiscale de l'amende}

Les limites que la loi donne au degré de sévérité de la peine d'amende applicable à une corporation ne se retrouvent pas toutes dans le texte même qui crée l'infraction. Ainsi, de même que la loi de libération conditionnelle des détenus ${ }^{56}$ atténue statutairement le degré de sévérité des peines d'emprisonnement de plus de deux ans imposées aux personnes physiques, tel qu'il fut interprété par la Cour fédérale du Canada ${ }^{57}$, vient atténuer l'importance des amendes imposées aux entreprises pour certaines sortes d'infractions.

En effet, aussi surprenant que cela puisse paraître, le juge Dubé de la division de première instance de la Cour fédérale du Canada, a rendu le 27 octobre 1976 dans la cause Day and Ross Ltd vs The Queen ${ }^{58}$ un jugement permettant à une compagnie de déduire des amendes encourues, aux fins de l'impôt, comme étant des dépenses faites pour produire un revenu.

Voici le résumé des faits de la cause donné dans le jugement :

The plaintiff company engaged in trucking business, had to pay fines for the violation of the provincial highway weight restriction laws. In computing its income, the company deducted the fines. The Minister disallowed the fines contending that their deduction was contrary to public policy. The company appealed contending that the deductions were expenses incurred for the purpose of producing income and were deductible pursuant to 121 (1) a of the former Act (Income Tax Act R.S.C. 1952) ${ }^{59}$.

55. Dumont, H. " La protection juridique du voisinage et de l'environnement à travers le droit pénal canadien ", Rapport québécois aux journées de l'Association Henri Capitant, Paris-Bordeaux 28 mai au 2 juin 1976, p.7, citant D. Trezise, " Alternative Approaches to Legal Control of Environmental Quality in Canada " 1975 Mc Gill L.J. 407.

56 S.R.C. (1970) Ch. P-2.

57 S.C. 19-20-21 Eliz.II, Ch.63.

58 (1976), D.T.C. 6433.

59. (1976), D.T.C. 6433 
Et voici l'essentiel de la décision de $M$. le juge Dubé :

In my view, the fines paid by the plaintiff in the case before me resulted from the day to day operation of its transport business and were paid as a necessary expense.

In the absence of constant control by the plaintiff over the exact cargo weight carried in its trailers, and the uncontradicted evidence would suggest that such a tight control would be impractical if not impossible in a very highly competitive road transport industry, then unintentional violations of weight restrictions would seem to be inevitable. Plaintiff's method of book-keeping, with fines paid entered as expense and fines recovered from customers booked as revenue, would also indicate that the payment of fines was very much a current item in the operation of plaintiff's business. The ready availability of advance over-weight permits at the request of a shipper would also tend to show that weight restrictions can be easily overcome and that violations thereof are obviously not outrageous transgressions of public policy ${ }^{60}$.

M. le juge Dubé semble donc vouloir s'opposer essentiellement aux deux arguments du Ministre du Revenu, à savoir que ces amendes ne sont pas des dépenses faites pour produire un revenu et qu'une telle déduction serait contraire à l'ordre public, en disant que l'amende résulte de l'opération quotidienne de l'entreprise de transport du contribuable et était une dépense nécessaire et que ces déductions ne sont pas contraires à l'ordre public, car les amendes ne furent pas imposées pour punir des transgressions odieuses de l'ordre public. Point n'est besoin d'imaginer la consternation des autorités gouvernementales devant un pareil jugement. On peut y apporter avec M. Neil Brooks, l'objection incluse dans le principe d'interprétation des lois, selon lequel autant que possible une loi ne doit pas être interprétée de manière à empêcher l'application des autres lois.

In the same way, if the courts and the legislatures are viewed as being engaged in a cooperative venture of law-making, a specific statute should be construed, if possible, so as not to frustrate the objectives of other statutes. This is a well recognized canon of statutory interpretation. There would appear to be no reason why this canon should be invoked only when construing specific words ${ }^{61}$.

Il découle de ce principe que ces déductions d'amendes ne devraient pas être admises dans la mesure où elles constituent une en-

60. Id., p. 6440.

61. Neil Brooks, Commentaire de l'arrêt Day and Ross V.R., (1977), 25 Can. Tax J., p. 18. 
trave à l'application d'autres lois et à l'obéissance spontanée aux dispositions de ces dernières. $\mathrm{M}$. Brooks analyse ainsi cette entrave :

What are the policy reasons for prohibiting the deductions of fines and penalties? The reasoning supporting such a prohibition must be that the deduction fo fines, in computing taxable income, would reduce the impact of the penalty and thus frustrate the intented deterrent effect of the fine. This reason for non-deductibility rests on at least two assumptions. First, if the deduction were allowed, the taxpayer would consider the tax savings as a factor in deciding whether the risk of noncompliance with the law outweighed the gains. Second, the legislative body which prescribed the fine did not consider that the sting would be reduced in some cases where it could be converted into a tax deduction. As empirical judgments, both assumptions are probably correct. Also, in the interests of law-making comity, the courts should no question at least the first assumption ${ }^{62}$.

Mais il se pourrait que la décision du juge Dubé soit véritablement fondée sur le fait qu'il n'ait pas considéré l'amende imposée pour l'infraction à cette loi provinciale comme une vraie peine, de telle sorte que la question au centre de ce jugement serait de savoir :

If the purpose of a fine for violation of weight restrictions on highways intended to deter drivers from overloading trucks, or is it simply a tariff or toll that the driver must pay, perhaps to cover additional wear and tear to the highways, for driving an overloaded truck? ${ }^{63}$

Mais cela n'est pas apparent à la lecture du jugement. Comme cette décision n'a pas fait l'objet d'un appel, ce jugement est devenu final, donnant ainsi un dur coup à toute velléité d'efficacité de la peine d'amende à l'égard des corporations et devenant une invitation à peine voilée à commettre des infractions pour produire du revenu. Car le juge Dubé va aussi loin que de dire qu'il est inévitable que la compagnie commette ces infractions à la loi pour pouvoir soutenir la concurrence commerciale.

... the uncontradicted evidence would suggest that such a tight control would be impractical if not impossible in a very highly competive road transport industry, then unintentional violations of weight restrictions would seem to be inevitable.

C'est là affirmer clairement la primauté de la réussite commerciale sur le respect de la loi.

Ainsi, à l'issue de cette partie consacrée à la gradation des

62. Neil Brooks, Commentaire de l'arrêt Day and Ross V.R., (1977). 25 Can. Tax J., p. 18.

63. Id., p. 19. 
montants d'amende on constate que cette gradation varie selon qu'il s'agit d'un acte criminel, d'une infraction punissable sur déclaration sommaire de culpabilité à une loi fédérale ou d'une infraction à une loi provinciale ou à un règlement municipal. Car s'il existe des règles générales pour régir l'importance des montants d'amende imposés aux corporations pour des infractions aux lois fédérales, il n'en existe pas pour les infractions aux lois du Québec. En effet, les peines applicables aux corporations peuvent varier selon les prescriptions spécifiques de chaque texte d'infraction dans les lois provinciales du Québec et dans les règlements des corporations municipales de cette province. Les peines prévues y sont alors généralement les mêmes pour les personnes physiques et pour les personnes morales.

Et si les peines d'amende pour un acte criminel peuvent atteindre virtuellement un degré très élevé de sévérité, la majorité des peines d'amende prévues pour une infraction punissable sur déclaration sommaire de culpabilité ne peuvent dépasser le montant de $1000 \$$. De plus ces dernières peuvent être atténuées par une récupération fiscale de l'amende, si celle-ci est encourue comme une dépense nécessaire pour produire un revenu.

\section{BIBLIOGRAPHIE}

"A Bill would overhaul U.S. laws ", The Montreal Star, 27 janvier 1978 A13.

BRAZIER, R. (1977) : "Appelate Attitudes Towards Compensation Orders ", Criminal Law Review, p. 712.

BRISSETTE, M. (1977) : L'infraction dans le droit pénal du Québec, Vol. I et II, Rapport final, Montréal, Université de Montréal, Centre international de criminologie comparée.

BRISSETTE, M. (1978) : Les sanctions pénales et les personnes morales, Mémoire de maîtrise en droit, Montréal, Université de Montréal, Faculté de Droit.

BROOKS, N. (1977) : "Commentaire de l'arrêt Day and Ross ", Canadian Tax Journal, vol. 25. p. 18.

CANADA. COMMISSION DE REFORME DU DROIT (1969) : La responsabilité stricte, Document de travail no 2, Ottawa, Information Canada.

CANADA. COMMISSION DE RÉFORME DU DROIT (1976) : Responsabilité pénale et conduite collective, Document de travail no 16, Ottawa, Information Canada.

DERSHOWITZ, A.M. (1961): Some Obseryations on the Meaning of Corporate Criminal Liability, Excerpts from a paper submitted by the author during his third term as a student in the Yale Law School.

HUNT, (1936) : The Development of the Business Corporation in England, London.

LEIGH, L.H. (1969) : The Criminal Liability of Corporation in English Law, L.S.E. Research Monographs, 2. London, Weidenfeld and Nicolson. 
McLEAN, I. (1973) : "Compensation and Restitution Orders ", Criminal Law Review. p.4.

RAYNAULD, A. (1964) : Institutions économiques canadienne, Montréal, Ed. Beauchemin.

SENEY, H.W. (1971) : «Harm, Danger and Dangerousness in Our Criminal Law », Wayne Law Review, vol. 17. p. 1111.

SENEY, H.W. (1971) : "Our Criminal Law's Moral Obsolescence ", Wayne Law Review, vol. 17. p. 777

WILlIAMS, G. (1961) : Criminal Law, The General Part, 2nd ed. London, Stevens. 